In press - British Journal of developmental Psychology

\title{
Childhood anxiety and attention to emotion faces in a modified Stroop task
}

Julie A. Hadwin ${ }^{1 *}$, Nick Donnelly ${ }^{2}$, Anne Richards ${ }^{3}$, Christopher C. French ${ }^{4}$ and Umang Patel ${ }^{1}$.

Running head: Childhood anxiety, social concern, inhibition, angry faces

*Corresponding author, Email: jah7@soton.ac.uk

${ }^{1}$ Developmental Brain-Behaviour Laboratory and the ${ }^{2}$ Centre for Visual Cognition

School of Psychology,

University of Southampton,

Southampton SO17 1BJ, U.K.

${ }^{3}$ Department of Psychology

Birkbeck College, University of London

Malet Street

London WC1E 7HX, U.K.

${ }^{4}$ Department of Psychology

Goldsmiths College, University of London,

New Cross

London SE14 6NW, U.K. 
This study used an emotional face stroop task to investigate the effects of self-report trait anxiety, social concern and chronological age on reaction time to match coloured outlines of angry, happy and neutral faces (and control faces with scrambled features) with coloured buttons in a community sample of 74 children aged six to twelve years. The results showed an interference of colour matching for angry (relative to neutral) faces in children with elevated social concern. The same effect was not found for happy or control faces. In addition, the results suggest that selective attention to angry faces in children with social concern was not significantly moderated by age. 


\section{Childhood anxiety and attention to emotion faces in a modified Stroop task}

Information processing models have proposed that individuals with elevated anxiety (Bar-Haim, Lamy, Bakermans-Kraneburg, Pergamin \& van IJzendoorn, 2007) or social anxiety (Clark \& McManus, 2001) selectively attend to threatening or negative stimuli. Bar-Haim and colleagues, for example, used a meta-analysis of the anxiety and attention literature to propose a theoretical framework. They suggest that anxious individuals evaluate and allocate their attention towards mildly threatening stimuli, where evaluation is suggested to occur preattentively. Research with adults (Mogg, Garner \& Bradley, 2007) and children (e.g., Hadwin et al., 2003) has used angry emotion faces to explore attention to threat in anxiety. Adult research has shown findings consistent with Bar-Haim et al.'s (2007) model to highlight links between increased trait anxiety and decreased reaction times (from now on RTs) to find angry (compared with happy) faces in visual search tasks (e.g., Byrne \& Eysenck, 1995). Further studies have highlighted a comparable attentional bias towards angry and fearful faces in a visual dot probe task in adults with elevated trait anxiety (Mogg et al., 2007).

Consistent with the framework proposed by Clark \& McManus (2002), a similar pattern of results has also been found in adults with elevated social anxiety. In adults with social phobia, studies have demonstrated faster RTs to find angry faces using visual search tasks (e.g., Gilboa-Schechtman, Foa \& Amir, 1999) and to detect a probe when preceded by angry versus happy faces in a visual dot probe task (Mogg, Philippot \& Bradley, 2004). Findings in the adult literature have been broadly supported using neuropsychological techniques. Eye movement studies, for example, have highlighted that adults with social phobia show "hyperscanning" (p.44) when viewing angry faces, indicating increased vigilance for threat (Horley, Williams, 
Gonsalvez \& Gordon, 2004). Adults with generalised anxiety disorder also showed faster RTs to locate angry faces measured using eye movements in a visual dot probe task (Mogg, Millar \& Bradley, 2000). In addition, socially phobic adults showed increased N170 amplitudes when categorising angry faces (Kolassa \& Miltner, 2006) and social anxiety symptoms in adults were associated with greater right amygdala activation in response to viewing "harsh" (angry, fearful, disgust) emotional faces (Phan, Fitzgerald, Natah \& Tancer, 2006).

Theory and research in the anxiety literature suggest that adults with generalised anxiety (including elevated trait anxiety) and social anxiety demonstrate attentional biases towards threat. Anxiety typically has its origins in childhood and can follow a chronic course through to adulthood (Cartwright-Hatton, McNicol, \& Doubleday, 2006). However, the volume of work in the developmental literature is limited. Consistent with information processing models of adult anxiety there is some evidence of attentional biases towards angry faces in children with elevated state and trait anxiety (Heim-Dreger, Kohlmann, Eschenbeck \& Burkhardt, 2006). Angry faces have also been found to facilitate (Hadwin et al., 2003) or impede (Ladouceur et al 2006) anxious children's performance on cognitive tasks. Furthermore, adolescents with elevated trait anxiety were more likely to interpret ambiguous faces as angry (Richards, French, Nash, Hadwin \& Donnelly, 2007). Most developmental studies have focused on the role of trait anxiety and attention to emotional faces. Fewer studies have explored social anxiety in children and attentional biases to threat. The results of one recent study suggested that typically developing children with elevated social anxiety showed some avoidance of angry and fearful faces (Stirling, Eley \& Clark, 2006). 
The aim of the present paper was to investigate selective attention to angry and happy faces in typically developing children with elevated trait and social anxiety. Kindt \& van den Hout (2001) proposed that attentional biases are evident in all young children and decline across childhood as a result of inhibitory processes that develop at around 10 years of age (Kindt, Brosschot \& Everaerd, 1997). Consequently, associations between attentional bias and individual differences in anxiety are expected to emerge around this time. Developmental studies typically focus on a limited age range to investigate attention to threat in childhood anxiety. One recent exception has found some evidence to support the developmental framework proposed by Kindt and van den Hout (2001). Perez-Olivas, Stevenson \& Hadwin (in press) found that children aged 10 years and over, with elevated separation anxiety, were faster to detect angry faces in a visual search task. This relationship was not found for children younger than ten years of age. A secondary aim of the current study was therefore to explore the role of age in attention to threat across childhood. It aimed to consider whether any relationship between anxiety and attention to threat was moderated by age, where this effect would be highlighted in a significant association between anxiety and attention to threat only in late childhood.

In order to explore attention to threat the current paper used an emotional stroop colour matching schematic face task. Schematic faces represent salient and unambiguous signals of emotion that (like real faces) elicit activation of the fusiform face area in imaging studies (Tong, Nakayama, Moscovitch, Weinrib \& Kanwisher, 2000). In addition, they allow the development of experimental control stimuli with scrambled facial features to explore whether the processing of angry faces is configural or feature-based (see Fox et al., 2000). If anxiety effects are shown for faces and not for control stimuli with scrambled facial features, then this would 
suggest that emotional faces and not individual component face features explain this pattern of results.

\section{Method}

\section{Participants}

Permission for this study was given by the Ethics Committee of the School of Psychology, University of Southampton, UK. Seventy-four children aged 6 to 12 years (mean age $=109$ months, $S D=23.871$, range $=6$ years; 2 months to 12 years; 10 months, 40 girls and 34 boys) took part in the study. The children represent a nonreferred population and were taken from three U.K. primary schools and one secondary school. In three schools $19 \%$ of parents provided informed written consent for their child to take part in the study. In the fourth school, parents were sent information letters and were given the opportunity to opt out of the study.

\section{Materials}

Revised Children’s Manifest Anxiety Scale (RCMAS) (Reynolds \& Richmond, 1985). The RCMAS, subtitled "What I Think and Feel," is a widely used 37-item self-report questionnaire that provides a measurement of trait anxiety and includes subscales scores related to social concern/concentration, physiological anxiety and worry/oversensitivity. RCMAS trait anxiety generates a total scaled score with a mean of 50 and SD of 10. Each subscale gives a scaled score with a mean of 10 and SD of 3. The trait anxiety and subscale scores have been shown to have good psychometric properties including moderate to high reliability (internal consistency and test-retest) and validity (construct and convergent and divergent; Reynolds and Richmond, 1985). Recent studies have also found that the trait anxiety and social concern subscale scores correlate with scores on more recently developed scales providing evidence of 
convergent validity (Muris, Merckelbach, Ollendick, King \& Bogie, 2002). Muris and colleagues showed that the RCMAS trait anxiety score correlated highly with the total score for the Multidimensional Anxiety Scale for Children (MASC; March, Parker, Sullivan Stallings and Connor, 1997). In addition, they highlighted that subscales of the MASC (physical symptoms, social anxiety, harm avoidance and separation anxiety) corresponded meaningfully to those of the RCMAS. In this case, the RCMAS social concern subscale was correlated with the MASC social anxiety subscale.

Apparatus. The stimuli were presented on a laptop computer (with a 12.1” flat visible screen) using the Superlab programming environment (Cedrus Corporation, 1996). The responses were made using the four central keys of a Superlab 6-key response box coloured red, blue, green and yellow from left to right.

Stimuli. Angry, happy, and neutral face stimuli made up the schematic faces, with each face being made up of a pair of eyes, eyebrows and a mouth (see Hadwin et al., 2003). The facial features from each emotion face were re-arranged to make control stimuli with scrambled facial features. Facial features were shown in white and face outline was red, blue, green or yellow. The presentation screen was black. Children saw 24 trials for each emotion; 12 emotion face and 12 scrambled face trials, making a total of 72 randomly presented trials. Face and scrambled stimuli were presented individually and in the same position on the screen.

\section{Procedure}

Children were seen individually in a quiet room or area outside their classroom and were asked to match the outline colour of a picture on the screen to the coloured buttons. Following this task, the RCMAS was completed. Children sat approximately $60 \mathrm{~cm}$ away from the computer screen with the response box on the 
desk in front of them. At the end of the session, the children were verbally debriefed and any questions were answered.

\section{Results}

\section{Participant Characteristics.}

The mean RCMAS total anxiety and the social concern/concentration, physiological and worry/ oversensitivity subscale scores were 49.53 ( $S D=12.26$, range $=13$ to 74$)$ and $9.43(S D=2.99$, range $=4-16), 9.71(S D=3.16$, range $=2$ to 16) and $9.30(S D=3.29$, range $=3$ to 17$)$ respectively. Children's trait anxiety scores were significantly correlated with all RCMAS subscales and the three subscales all correlated with each other (in all cases $p<.01$, see Table 1.)

\section{Stroop Task}

Following Ratcliffe (1993) the reciprocal means of the RT data were used in the analysis, where these remove the influence of RT outliers without imposing arbitrary cut-offs on the data. For the purposes of reporting or manipulating RT data to create bias scores (see below) the reciprocal mean can be converted back to RT scores by calculating 1/reciprocal mean. The converted RT mean is used in the current paper for reporting and presentation of the data. The mean RTs for angry, happy, neutral faces and the three corresponding control faces with scrambled features were $644.81 \mathrm{~ms}, 670.44 \mathrm{~ms}, 690.32 \mathrm{~ms}$ and $689.93 \mathrm{~ms}, 683 \mathrm{~ms}, 679.92$ respectively. The respective mean percentage of correct responses was $97.78 \%, 98.11 \%, 98.17 \%$ and 98.42\%, 98.31\% and 97.86\% respectively.

For both the emotion and the control faces, attentional bias scores were calculated by subtracting individual mean RT values for neutral faces from those for angry and happy faces. This created angry-neutral and happy-neutral bias scores for 
emotion faces and for control faces; positive scores indicated interference to colour matching and negative scores indicated facilitation. One bias score outlier that was more than three standard deviations from the group mean was removed from the happy-neutral analysis. Pearson's correlations were carried out to look at the relationship between chronological age (CA), trait anxiety, social concern, physiological anxiety and worry/ oversensitivity with the angry-neutral and happyneutral bias scores for emotion and control faces. This analysis showed that increased social concern was associated with increased angry bias scores $(r=.24, \mathrm{p}<.05)$. There were no significant correlations between trait anxiety and the physiological or worry/ oversensitivity anxiety subscale scores with the angry or happy bias scores or with between anxiety measure and the control face bias scores. (in all cases $r<.10$, see Table 1).

In order to explore whether the association between angry bias scores and social concern (SC) was moderated by CA, we used multiple regression analysis. Standardised versions of the variables (z scores) were computed for SC and CA and a product term was created (by multiplying the two predictor variables together) to form an interaction variable (SC X CA). CA was entered on the first step. This produced a non-significant regression equation $(F(1,73)=1.98, \beta=-.16, p>.1)$. Social concern was entered on the next step producing a significant regression equation $(F(2,73)=$ 3.63, $p<.05 ; \beta$ for SC $=.26, p<.05$ ), accounting for $9 \%$ in the variance for the angry-neutral bias score. The product term, SC X CA, was entered on the third step but this did not result in a significant change to the model $\left(R^{2}=.03, \mathrm{p}>.10 ; \beta\right.$ for SC $\mathrm{X} \mathrm{CA}=.12, p>.10$ ), accounting for $12 \%$ of the variance. Further analysis that divided children into two age groups (6-8 years vs. 9-12 years) confirmed that age group did not significantly moderate the SC effect on angry-neutral bias scores. In 
addition, no significant effects of anxiety scores or age were observed in similar analyses of the happy-neutral and control face bias scores

\section{Discussion}

The present study employed an emotional Stroop schematic face task to demonstrate that colour-matching interference for angry (relative to neutral) faces was associated with higher levels of social concern in children aged 6 to 12 years. The results are consistent with adult models of anxiety (e.g., Bar-Haim et al., 2007; Clark \& McManus, 2001) in demonstrating links between individual differences in social concern in children and attention to threatening stimuli. The results support findings in the adult anxiety literature which have found links between elevated or clinical social anxiety and selective attention to angry faces (e.g., Horley et al., 2004; Mogg et al., 2004). The results from the present study regarding experimental control stimuli with scrambled facial features suggest that children process emotion faces configurally and highlight one advantage of using schematic faces in developmental research.

Failure to inhibit attention to angry faces in the present paper was specific to increased social concern, raising the possibility that the developmental picture related to information processing biases in childhood anxiety can be most clearly understood by matching specific anxieties with relevant experimental stimuli (Pine et al., 2005). The presence of information processing biases in children with elevated social concern across this age range has implications for understanding risk factors associated with the emergence of social anxiety disorder in late childhood (Essau, Conradt \& Petermann, 2000). It is possible, for example, that the presence of 
information processing biases in middle childhood represent a risk factor for the development of anxiety in late childhood or early adolescence.

The results did not fit well with the developmental framework outlined by Kindt \& van den Hout (2001), which suggests that individual differences in anxiety should come to be associated with attentional bias only in late childhood. Kindt's empirical work to support this framework did, however, use different stimuli; spider words and not emotional faces were used to explore attentional biases in children aged eight to twelve years (see Kindt et al., 1997). However, Perez-Olivas et al., (in press) did find evidence for decreased RTs to find angry faces in a visual search task in children with increased separation anxiety in late childhood. Taken together, these findings suggest that age related change may be apparent only for certain types of phobia. It is also possible that the emotional face stroop paradigm is more sensitive in identifying attentional biases in young socially anxious children compared to a visual search for emotional faces. Future studies should aim to investigate developmental differences in attentional biases across different attentional paradigms, using a broader age range and with increased numbers in each age group.

In summary, the results of the present study demonstrated that increased social concern in children from six to twelve years of age was associated with decreased ability to inhibit attention to angry faces and that this relationship was not moderated by age. However, it is possible that age related change is a function of different aspects of anxiety or may be more demonstrable using experimental paradigms that tap different aspects of attention. Longitudinal data is needed to understand whether attentional biases to threat in typically developing groups of children pose a risk factor for the development of anxiety disorders in late childhood or in adolescence. 


\section{References}

Bair-Haim, Y., Lamy, D., Pergamin, L., Bakermans-Kranenburg, M.J. \& van IJzendoorn, M.H. (2007). Threat-related attentional bias in anxious and nonanxious individuals: A meta-analytic study. Psychological Bulletin, 133, 1124.

Byrne, A. \& Eysenck, M.W. (1995). Trait anxiety, anxious mood and threat detection. Cognition and Emotion, 92 549-562.

Cartwright-Hatton, S., McNicol, K., \& Doubleday, E. (2006). Anxiety in a neglected population: Prevalence of anxiety disorders in pre-adolescent children. Clinical Psychology Review, 26, 817-833.

Cedrus Corporation (1996). Superlab Pro. San Pedro, CA, USA: Cedrus Corporation.

Clark, D.M. \& McManus, F. (2002). Information processing in social phobia. Biological Psychiatry, 51, 92-100.

Essau, C., Conradt, J., \& Petermann, F. (2000). Frequency, co-morbidity and psychosocial impairment of anxiety disorders in adolescents. Journal of Anxiety Disorders, 14, 263-279.

Fox, E., Lester, V., Russo, R., Bowles, R.J., Pichler, A. \& Dutton, K. (2000). Facial expression of emotion: Are angry faces detected more efficiently? Cognition and Emotion, 14, 61-92.

Hadwin, J.A., Donnelly, N., French, C.C., Richards, A., Watts, A., \& Daley, D. (2003). The influence of children's self-report trait anxiety and depression on visual search for emotional faces. Journal of Child Psychology and Psychiatry, 44, 432-444. 
Heim-Dreger, U., Kohlman, C., Eschenbeck, H. \& Burkhardt, U. (2006). Attentional biases for threatening faces in children: Vigilant and avoidant processes. Emotion, 6, 320-325.

Horley, K., Williams, L.M., Gonsalvez, C., \& Gordon, E. (2004). Face to face: visual scanpath evidence for abnormal processing of facial expressions in social phobia. Psychiatry Research, 127, 43-53.

Kindt, M. \& Van Den Hout, M. (2001). Selective attention and anxiety: A perspective on developmental issues and the causal status. Journal of Psychopathology and Behavioural Assessment, 23, 193-202.

Kindt, M., Bierman, D. \& Brosschot, J. F. (1997). Cognitive bias in spider fear and control children: Assessment of emotional interference by a card format and a single-trail format of the stroop task. Journal of Experimental Child Psychology, 66, 163-179.

Kolassa, I-T. \& Miltner, W.H.R. (2006). Psychophysiological correlates of face processing in social phobia. Brain research, 1118, 130-141.

Ladouceur, C.D, Dahl, R.E., Williamson, D.E., Birmaher, B., Axelson, D.A., Ryan, N.D. \& Casey, B.J. (2006). Processing emotional facial expression influences performance on a Go/NoGo task in pediatric anxiety and depression. Journal of Child psychology and Psychiatry, 47, 1107-1115.

March, J.S., Parker, J.D., Sullivan, K., Stallings, P. \& Connors, C. (2002). Correlations between two multidimensional anxiety scales for children. Journal of the American Academy of Child and Adolescent Psychiatry, 36, 554-565.

Mogg, K., Garner, M. \& Bradley, B.P. (2007). Anxiety and orienting of gaze to angry and fearful faces. Biological Psychiatry. 
Mogg, K., Philipot, P. \& Bradley, B.P. (2004). Selective attention to angry faces in clinical social phobia. Journal of Abnormal Psychology, 113, 160-165.

Mogg, K., Millar, N \& Bradley, B.P. (2000). Biases in eye movements to threatening facial expressions in generalised anxiety disorder and depressive disorder. Journal of Abnormal Psychology, 109, 695-704.

Muris, P., Merckelbach, H., Ollendick, T., King, N. \& Bogie, N. (2002). Three traditional and three new childhood anxiety questionnaires: their reliability and validity in a normal adolescent sample. Behaviour research and Therapy, 40, 753-772.

Öhman, A., Lundquvist, D. \& Esteves, F. (2001). The face in the crowd revisited: A threat advantage with schematic stimuli. Journal of Personality and Social Psychology, 80, 381-396.

Phan, K.L., Fitzgerald, D.A., Pradeep, J.N. \& Tancer, M.E. (2006). Association between amygdala hyperactivity to harsh faces and severity of social anxiety in generalised social phobia. Biological Psychiatry, 59, 424-429.

Perez-Olivas, G., Stevenson, J. \& Hadwin, J.A. (in press). Do anxiety-related attentional biases mediate the link between maternal over involvement and separation anxiety in children? Cognition and Emotion.

Pine, D.S., Klein, R.G., Mannuzza, S., Moulton, J.L., Lissek, S., Guardino, M., \& Woldehawariat, G. (2005). Face-emotion processing in offspring at risk for panic disorder. Journal of the American Academy of Child and Adolescent Psychiatry, 44, 664-672.

Ratcliffe, R. (1993). Methods for dealing with reaction time outliers. Psychological Bulletin, 114(3), 510-532. 
Reynolds C.R. \& Richmond, B.O. (1985) Revised Children's Manifest Anxiety Scale (RCMAS) Western Psychological Services.

Richards, A., French, C.C., Nash, G., Hadwin, J.A. and Donnelly, N. (2007). A comparison of selective attention and facial processing biases in typically developing children high and low in self-reported trait anxiety. Development and Psychopathology, 19, 481-495.

Stirling, L.J., Eley, T.C. and Clark, D.M. (2006). Preliminary evidence for an association between social anxiety symptoms and avoidance of negative faces in school-age children. Journal of Clinical Child and Adolescent Psychology, 35, 440-445.

Tong, F., Nakayama, K., Moscovitch, M., Weinrib, O. and Keinwischer, N. (2000). Cognitive Neuropsychology, 17, 257-279. 


\section{Table 1.}

Pearson's correlations between chronological age (CA), trait anxiety, social concern, physiological anxiety and worry/ oversensitivity with angry-neutral and happy-neutral emotion and control (scrambled face) bias scores.

\begin{tabular}{|c|c|c|c|c|c|c|c|c|c|}
\hline Variables & 1. & 2. & 3. & 4. & 5. & 6. & 7. & 8. & 9. \\
\hline 1. CA & -- & .02 & -.04 & -.02 & .08 & -.16 & -.06 & -.03 & -.09 \\
\hline 2. Trait anxiety & & -- & $.83^{* *}$ & $.88^{* *}$ & $.89^{* *}$ & .19 & .01 & .01 & -.01 \\
\hline 3. Social concern & & & -- & $.67^{* *}$ & $.67^{* *}$ & $.26^{*}$ & .05 & -.03 & -.09 \\
\hline 4. Physiological anxiety & & & & -- & $.66^{* *}$ & .15 & -.02 & .01 & .03 \\
\hline 5. Worry/oversensitivity & & & & & -- & .12 & .04 & .02 & .04 \\
\hline 6. Angry-neutral face bias & & & & & & -- & $.51^{* *}$ & -.01 & -.09 \\
\hline 7. Happy-neutral face bias & & & & & & & -- & $.51^{* *}$ & .07 \\
\hline 8. Angry-neutral control bias & & & & & & & & -- & $.36^{*}$ \\
\hline 9. Happy-neutral control bias & & & & & & & & & -- \\
\hline
\end{tabular}


